\title{
Implementation of Korean Clinical Imaging Guidelines: A Mobile App-Based Decision Support System
}

\author{
Jeong Hoon Lee, $\mathrm{PhD}^{1}$, Eun Ju Ha, MD, PhD², Jung Hwan Baek, MD, PhD², \\ Miyoung Choi, RN, MPH, $\mathrm{PhD}^{4}$, Seung Eun Jung, MD, PhD ${ }^{5}$, Hwan Seok Yong, MD, $\mathrm{PhD}^{6}$
}

${ }^{1}$ Division of Biomedical Informatics, Seoul National University Biomedical Informatics (SNUBI), Seoul National University College of Medicine, Seoul, Korea; ${ }^{2}$ Department of Radiology, Ajou University School of Medicine, Suwon, Korea; ${ }^{3}$ Department of Radiology and Research Institute of Radiology, University of Ulsan College of Medicine, Asan Medical Center, Seoul, Korea; 'Division for Healthcare Technology Assessment Research, National Evidence-Based Healthcare Collaborating Agency, Seoul, Korea; ${ }^{5}$ Department of Radiology, Seoul St. Mary's Hospital, College of Medicine, The Catholic University of Korea, Seoul, Korea; ${ }^{6}$ Department of Radiology, Korea University Guro Hospital, Korea University College of Medicine, Seoul, Korea

Objective: The aims of this study were to develop a mobile app-based clinical decision support system (CDSS) for implementation of Korean clinical imaging guidelines (K-CIGs) and to assess future developments therein.

Materials and Methods: K-CIGs were implemented in the form of a web-based application (http://cdss.or.kr/). The app containing K-CIGs consists of 53 information databases, including 10 medical subspecialties and 119 guidelines, developed by the Korean Society of Radiology (KSR) between 2015 and 2017. An email survey consisting of 18 questions on the implementation of K-CIGs and the mobile app-based CDSS was distributed to 43 members of the guideline working group (expert members of the KSR and Korean Academy of Oral and Maxillofacial Radiology) and 23 members of the consultant group (clinical experts belonging to related medical societies) to gauge opinion on the future developmental direction of K-CIGs. Results: The web-based mobile app can be downloaded from the Google Play Store. Detailed information on the grade of recommendation, evidence level, and radiation dose for each imaging modality in the K-CIGs can be accessed via the home page and side menus. In total, 32 of the 66 experts contacted completed the survey (response rate, 45\%). Twenty-four of the 32 respondents were from the working group and eight were from the consulting group. Most $(93.8 \%)$ of the respondents agreed on the need for ongoing development and implementation of K-CIGs.

Conclusion: This study describes the mobile app-based CDSS designed for implementation of K-CIGs in Korea. The results will allow physicians to have easy access to the K-CIGs and encourage appropriate use of imaging modalities.

Keywords: Medical cost; Imaging modality; Health insurance system; Healthcare expense

\section{INTRODUCTION}

Evidence-based medicine (EBM) is characterized by conscientious, explicit, and judicious use of the best available evidence when making decisions about the care of individual patients (1). In clinical practice, EBM refers to the integration of clinical expertise with the best available external clinical evidence, as derived from systematic research, coupled with consideration of each patient's individual values, to inform the decision-making process regarding patient care (1-3). In the era of EBM, clinical practice guidelines, which are systematically developed statements used to assist practitioners and patients regarding decisions on appropriate treatments under specific

Received September 15, 2018; accepted after revision September 16, 2018.

This study was supported by a grant of the Korea Health Technology R\&D Project through the Korea Health Industry Development Institute (KHIDI), funded by the Ministry of Health \& Welfare, Republic of Korea (grant number: HC17C0092).

Corresponding author: Eun Ju Ha, MD, PhD, Department of Radiology, Ajou University School of Medicine, 206 World cup-ro, Yeongtonggu, Suwon 16499, Korea.

- Tel: (8231) 219-4057•Fax: (8231) 219-5852•E-mail: radhej@naver.com

This is an 0pen Access article distributed under the terms of the Creative Commons Attribution Non-Commercial License (https:// creativecommons.org/licenses/by-nc/4.0) which permits unrestricted non-commercial use, distribution, and reproduction in any medium, provided the original work is properly cited. 
clinical circumstances, are essential to optimize patient care and to assess the benefits and drawbacks of alternative treatment options $(3,4)$.

In the field of radiology, overuse or inappropriate use of imaging modalities is a long-standing issue because of the potential harm caused by unnecessarily exposing patients to excess radiation, as well as increased medical costs (59). However, ordering appropriate imaging tests can be challenging for many physicians, particularly under time constraints and when there is no clear guideline to support a decision. Therefore, many countries have started to develop clinical imaging guidelines (CIGs) to determine the most appropriate imaging modalities based on the best available evidence (10-12). These CIGs are also publicly available as mobile applications in the form of clinical decision support systems (CDSSs), which can be easily accessed by ordering physicians and their patients (11-13). Use of CIGs is believed to enhance quality of care and efficacious use of radiology (7).

The Korean Society of Radiology (KSR) started development of Korean CIGs (K-CIGs) in 2015, and work thereon is still in progress $(14,15)$. In response to the increasing number of guidelines, a mobile app-based CDSS was proposed via which K-CIGs could be implemented. The aims of this study were to develop a mobile app-based CDSS for implementation of K-CIGs and to assess future developments therein.

\section{MATERIALS AND METHODS}

\section{Planning and Development of a Mobile App-Based CDSS}

A web-based application was designed to promote clinical implementation of K-CIGs. Angular JS, which is a JavaScriptbased, dynamic web application platform, and the Firebase database and Firebase-Auth frameworks, which constitute a mobile app platform and cloud-based, real-time database (Google, Inc., San Jose, CA, USA; https://firebase.google. $\mathrm{com} /$ ), were used to create a data storage facility for the mobile web application interface and Android application $(16,17)$. This service includes a real-time NoSQL database management system with guaranteed scalability of data.

The app allows keyword searches in the K-CIGs, based on symptoms and diseases, thereby allowing easier access to guidelines for ordering physicians and patients. It also groups the guidelines according to 10 subspecialties (brain/head and neck, thyroid, chest, cardiovascular, breast, abdomen, genitourinary, musculoskeletal, pediatric, and interventional). A total of 119 guidelines for 53 key questions were integrated into a single JavaScript Object Notation (JSON) format using the jsonlite package of $R$ software, which uses a tabular relationship instead of traditional relational databases (18). The K-CIGs can be downloaded as a JSON file via the "About CDSS" menu without authorization.

\section{The Survey}

An email survey comprising 18 questions on implementation of the K-CIGs was distributed to 43 expert members of the KSR and the Korean Academy of Oral and Maxillofacial Radiology and to 23 clinical experts belonging to related medical societies (consultant group) in January 2018. The survey aimed to gauge opinion on the current status and future developmental direction of K-CIGs (Table 1). Most of the questions were answered on a 7-point scale. The questions were organized into five domains: awareness of developments in K-CIGs; obstacles to application of K-CIGs; awareness of the development of a mobile appbased CDSS; the expected clinical utility of the app; and promotion of implementation of K-CIGs. Responses were received over a 2-week period and reminders were sent out twice during that time.

\section{RESULTS}

\section{Mobile App-Based CDSS}

K-CIGs were implemented as a web application (http:// cdss.or.kr/) and can be accessed by the public without the need for any special permissions. The web-based Android applications can also be downloaded from the Google Play Store.

Detailed information on the grade of recommendation, evidence level, and radiation dose for each imaging modality in the K-CIGs can be accessed via the home page and side menus (Fig. 1). Recommendation grades in the $\mathrm{K}$-CIGs can be classified by reliability from the most (grade A) to the least reliable (i.e., not recommended; grade C). Grade I indicates no recommendation. The level of evidence when grading each K-CIG was categorized as high (I), moderate (II), low (III), or very low (IV). The radiation level of the different imaging modalities was divided into five categories according to the likely risk of radiation exposure, measured in $\mathrm{mSv}$, and based on the methodology used to develop evidence-based CIGs (19) (Fig. 2). The values for all the information are visualized as a simple colored bar 


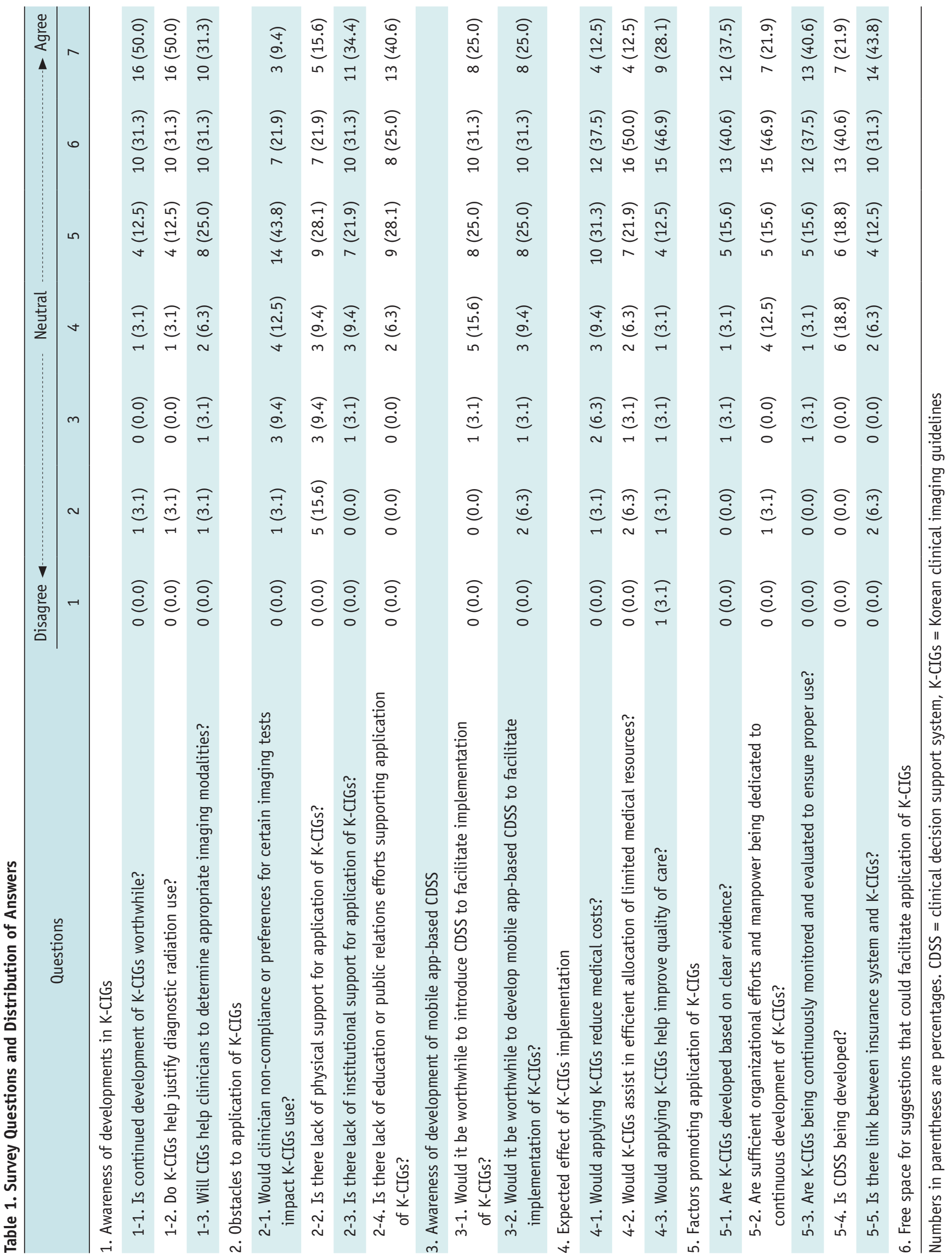




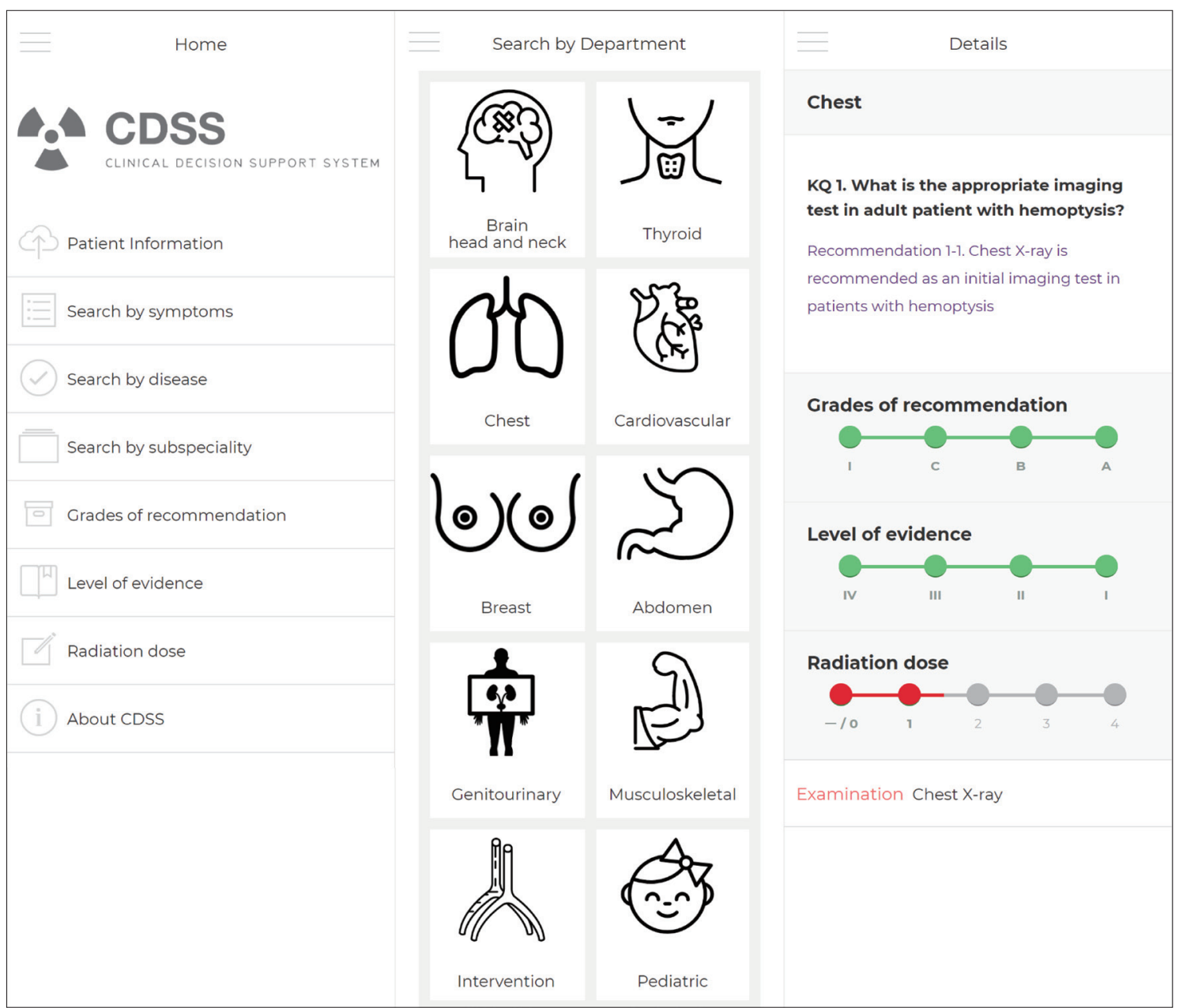

Fig. 1. Algorithm for mobile app-based CDSS. K-CIGs can be searched for using keywords (symptoms or diseases) or by 10 subspecialty categories (via home page and side menus). CDSS = clinical decision support system, K-CIGs = Korean clinical imaging guidelines

plot. The recommendation grades and levels of evidence are positive in the lower sense, so plots are visualized in the reverse direction with green color. Conversely, the radiation dose is drawn in the forward direction with red color in the same manner.

\section{The Survey}

The 18-question survey was sent to the 66 experts detailed above and completed by 32 respondents (response rate, $45 \%$ ). Twenty-four of the 32 respondents were from the working group and eight were from the consulting group (Fig. 3).

Table 1 shows the survey questions and the distribution of answers. Regarding awareness of developments in the K-CIGs, most of the respondents $(93.8 \%, 30 / 32)$ agreed on the need for continued development and implementation of K-CIGs; few respondents disagreed $(3.1 \%, 1 / 32)$ or were undecided $(3.1 \%, 1 / 32)$. Most $(93.8 \%, 30 / 32)$ agreed that developing K-CIGs was important to justify the use of diagnostic radiation, and $87.6 \%$ (28/32) answered that K-CIGs would help ordering clinicians to determine the appropriate imaging tools for a given case. However, $24 / 32$ respondents $(75.0 \%)$ answered that clinician noncompliance or preferences for particular imaging modalities would affect the application of K-CIGs, while 4/32 (12.5\%) considered that personal factors would have little effect on implementation of K-CIGs. In addition, 21/32 respondents (65.5\%) noted a lack of any computerized system integrated with electronic medical records and $28 / 32(87.5 \%)$ noted lack of an institutional environment, such as a health insurance or incentive system, to support application of K-CIGs. Meanwhile, 30/32 respondents (93.8\%) noted a lack of provision of educational programs or public relations strategies by the KSR to support application of K-CIGs.

Twenty-six of 32 respondents (81.3\%) agreed that introduction of a CDSS to support application of K-CIGs, 


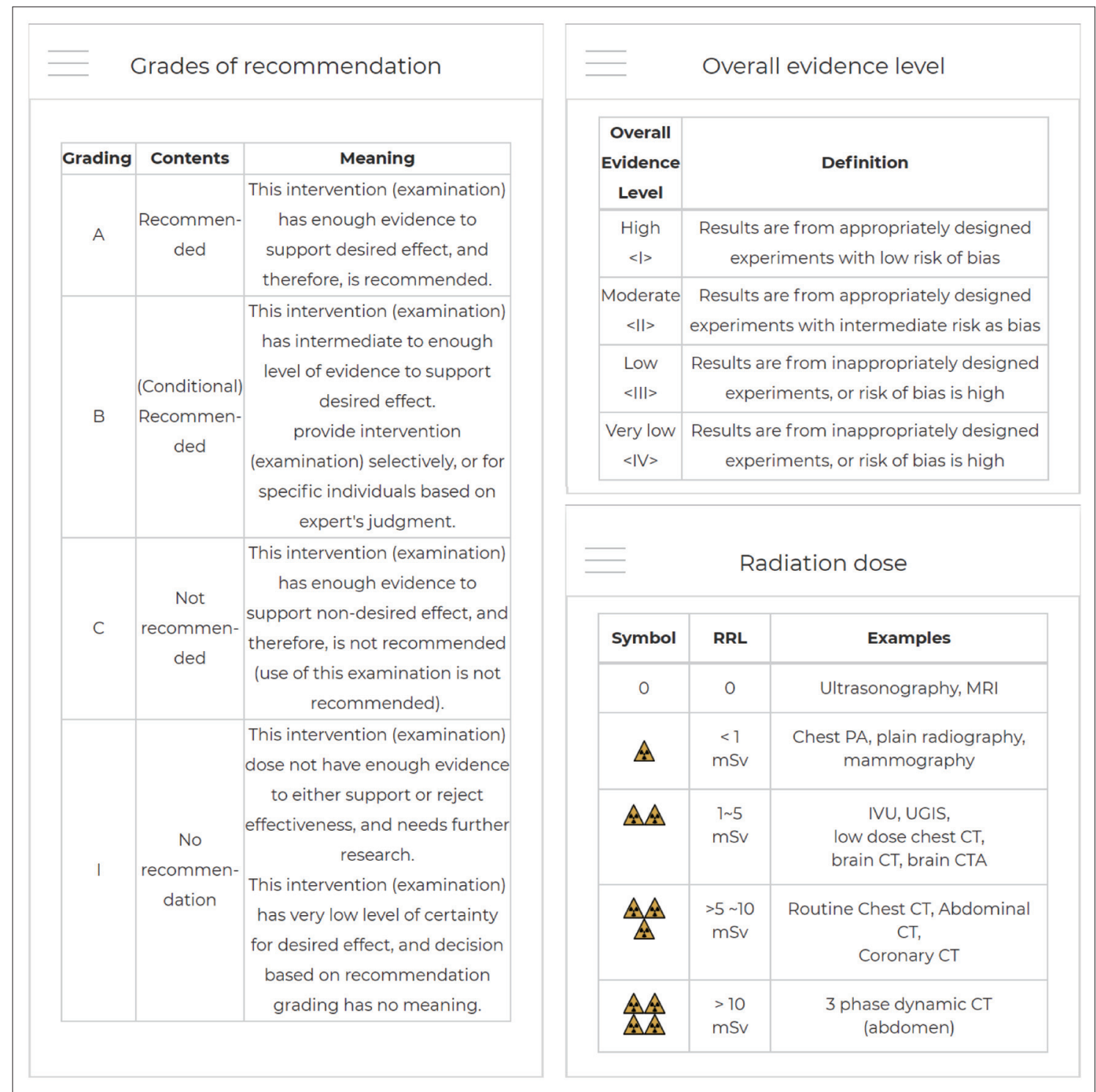

Fig. 2. Information on recommendation grade, evidence level, and radiation dose provided by K-CIGs. CT = computed tomography, $\mathrm{CTA}=$ computed tomography angiography, IVU = intravenous urography, $\mathrm{MRI}=$ magnetic resonance imaging, $\mathrm{PA}=$ posteroanterior, $\mathrm{RRL}=\mathrm{relative}$ radiation level, UGIS = upper gastrointestinal imaging series

including via a mobile app, would be worthwhile. Moreover, $26 / 32$ respondents $(81.3 \%)$ agreed that K-CIGs would contribute to reducing medical costs, while $27 / 32(84.4 \%)$ agreed that such an application would assist in efficient allocation of limited medical resources. In addition, $28 / 32$ respondents $(87.5 \%)$ agreed that K-CIGs would ultimately help to improve the quality of health care. In terms of promoting the use of K-CIGs, clear evidence-based guidelines (93.8\%, 30/32), continuous monitoring and evaluation of appropriate use $(93.8 \%, 30 / 32)$, a link with the health insurance system $(87.5 \%, 28 / 32)$, organizational efforts and manpower dedicated to development of K-CIGs (84.4\%, 27/32), and development of CDSS (81.3\%,
26/32) were all considered necessary by the majority of respondents. Continuous guideline updates to keep pace with emerging evidence, as well as promotional and educational activities aimed at clinicians and the general public, were also suggested by the respondents as directions for future development of K-CIGs.

\section{DISCUSSION}

Health care expenses are steadily increasing worldwide, and imaging studies account for a considerable proportion of all health care costs. However, although imaging is useful for accurate diagnosis and treatment, it may not 


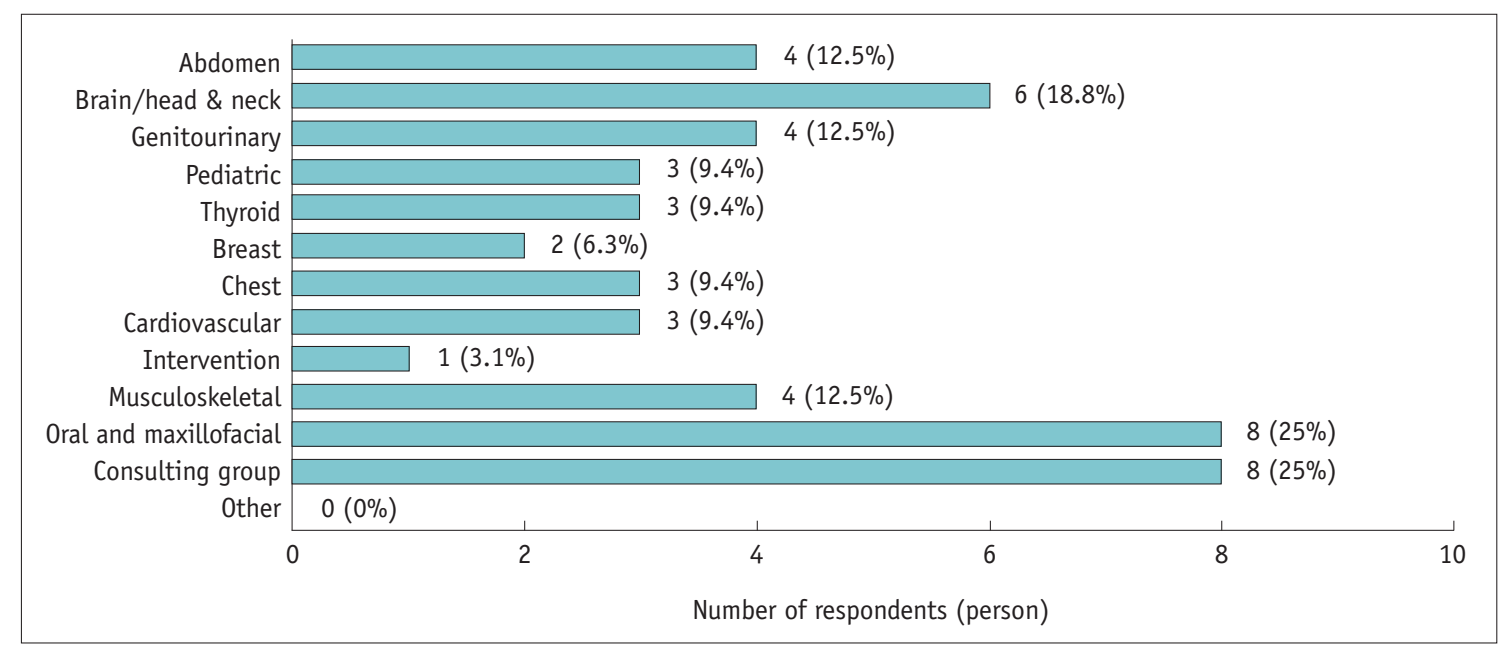

Fig. 3. Radiologic subspecialties represented by respondents (multiple responses possible).

be appropriate for certain conditions, and issues remain regarding the steady increase in exposure of the general population to medical radiation over the past 20 years (5-9). These issues are largely attributable to the use of computed tomography and nuclear medicine, and several studies have suggested that a percentage of all future cancers will result from current imaging use $(5,6)$. Therefore, judicious use of radiology is now a major issue, with a change in the prevailing concept from volume-based to value-based care in radiology (8). The American College of Radiology (ACR) Appropriateness Criteria are well-known evidencebased guidelines that assist referring physicians and other health care providers to make the most appropriate imaging decisions for specific clinical conditions (10). Since 1993, these guidelines have been continuously updated and have been integrated with electronic health/medical records within a CDSS known as the ACR Select ${ }^{\mathrm{TM}}$ (20). This resource covers more than 3000 clinical scenarios and includes 15000 clinical endpoints. A study by Huber et al. (21) reported a significant improvement in appropriateness scores, especially in imaging studies ordered by trainees, after implementation of a CDSS tool in the United States. Similarly, the Royal College of Radiologists have continually developed referral guidelines for imaging modalities (i.e., iRefer) since 1989 and has incorporated these guidelines into a CDSS known as OrderWise (MedCurrent Corp., Toronto, Canada), which affords simultaneous access to criteria for appropriate use and clinical guidelines from multiple sources (11). As part of these efforts, the European Society of Radiology (ESR) also provides the ESR iGuide (22).

In Korea, the development of K-CIGs began in 2015 based on the process of adapting existing imaging guidelines (19).
In the protocol for development of K-CIGs, 53 guidelines pertaining to 25 key questions were initially developed during 2015-2016 with support from the KSR and the National Evidence-based Healthcare Collaborating Agency (14). Sixty-six guidelines for 28 key questions were then developed during 2016-2017, again with support from the KSR and Korea Centers for Disease Control and Prevention (15). However, although paper records and searchable PDFs are useful, they have limitations with respect to use of K-CIGs at the point of care. Therefore, a web-based app was considered a necessity for clinical use of K-CIGs. A mobile app-based CDSS is the first step toward such clinical use and has potential for widespread uptake by ordering physicians. Moreover, it is expected that mobile app-based CDSS will contribute to efficacious use of radiology (7, $23,24)$. In the present survey, most respondents agreed regarding the need to develop and implement K-CIGs and approved of the introduction of a mobile app-based CDSS. However, for the development of such an app, obstacles such as clinician noncompliance or specific preferences would need to be addressed, and improvements in physical, institutional, and educational support would be required. In-depth discussions are necessary to determine the environmental factors most in need of improvement, and how these improvements could be achieved in the future. These efforts will assist physicians in appropriate utilization of imaging modalities. In particular, increasing the range of diagnostic imaging scenarios via ongoing refinements in expert-developed guidelines is necessary to promote the use of K-CIGs.

A CDSS may be defined as a computerized system designed to improve clinical decision-making pertaining to 
individual patients (25). A CDSS that assists in prescribing, diagnosis, therapy, information retrieval, and image analysis could take a number of forms. CDSS confers a number of important benefits by promoting avoidance of errors and thus an increase in the quality of care and, potentially, superior patient outcomes. In the field of radiology, several studies have reported on the potential impact of adoption and use of a computerized physician order entry system integrated with CDSS for imaging $(21,24)$. Such systems, if widely accepted for clinical use, would influence ordering habits and potentially increase the appropriateness of imaging studies, especially in emergency department and inpatient settings. Although many examinations are ordered without involvement of radiologists, we believe that a mobile app-based CDSS will be useful to guide physicians toward appropriate imaging test requests. In ACR Select ${ }^{\mathrm{TM}}$, the CDSS provides an appropriateness score for every examination; these data can then be used as the basis for analytics and are expected to assist in understanding the reasons for inappropriate utilization of imaging modalities (20). Radiologists could also contribute to education designed to improve the performance of ordering physicians. Such measures could have a positive impact on creation of $\mathrm{K}$-CIGs and their implementation in practice.

This study had some limitations. First, the proposed mobile app-based CDSS is not a final product, but rather the first step toward developing such a tool, which will ultimately be integrated into the medical records system. The system is expected to be continuously updated according to the newly developed guidelines. Second, although the survey was sent to all members of the guidelines working group and consulting group, the response rate was only $45 \%$. Therefore, there may be some degree of selection bias in the results.

In conclusion, this study proposes the first mobile appbased CDSS to facilitate implementation of K-CIGs in Korea. The results will allow physicians to have easy access to the K-CIGs and encourage appropriate use of imaging modalities.

\section{Conflicts of Interest}

The authors have no financial conflicts of interest.

\section{ORCID}

Eun Ju Ha

$$
\text { https://orcid.org/0000-0002-1234-2919 }
$$

Jeong Hoon Lee

https://orcid.org/0000-0002-1789-8270

\section{REFERENCES}

1. Sackett DL, Rosenberg WM, Gray JA, Haynes RB, Richardson WS. Evidence based medicine: what it is and what it isn't. BMJ 1996;312:71-72

2. Evidence-Based Medicine Working Group. Evidence-based medicine. A new approach to teaching the practice of medicine. JAMA 1992;268:2420-2425

3. Eddy DM. Practice policies: where do they come from? JAMA 1990;263:1265, 1269, 1272 passim

4. Lim W, Arnold DM, Bachanova V, Haspel RL, Rosovsky RP, Shustov AR, et al. Evidence-based guidelines--An introduction. Hematology Am Soc Hematol Educ Program 2008:26-30

5. Brenner DJ, Hall EJ. Computed tomography--An increasing source of radiation exposure. N Engl J Med 2007;357:22772284

6. Sodickson A, Baeyens PF, Andriole KP, Prevedello LM, Nawfel $\mathrm{RD}$, Hanson $\mathrm{R}$, et al. Recurrent $\mathrm{CT}$, cumulative radiation exposure, and associated radiation-induced cancer risks from CT of adults. Radiology 2009;251:175-184

7. Morgan TA, Avrin DE, Carr CD, Dreyer KJ, Flanders AE, Khorasani $R$, et al. Meaningful use for radiology: current status and future directions. Radiology 2013;269:318-321

8. Malone J, Guleria R, Craven C, Horton P, Järvinen H, Mayo $\mathrm{J}$, et al. Justification of diagnostic medical exposures: some practical issues. Report of an International Atomic Energy Agency Consultation. Br J Radiol 2012;85:523-538

9. Lehnert BE, Bree RL. Analysis of appropriateness of outpatient CT and MRI referred from primary care clinics at an academic medical center: how critical is the need for improved decision support? J Am Coll Radiol 2010;7:192-197

10. ACR Appropriateness Criteria. American College of Radiology Web site. http://www.acr.org/Quality-Safety/AppropriatenessCriteria. Published 2018. Accessed July 15, 2018

11. Making the best use of clinical radiology. iRefer Web site. http://www.irefer.org.uk. Accessed July 15, 2018

12. Diagnostic Imaging Pathways Web site. http://www. imagingpathways.health.wa.gov.au/index.php/about-imaging. Accessed July 15, 2018

13. Best practices guidelines on imaging clinical decision support systems. American College of Radiology Web site. https:// www.acr.org/-/media/ACR/Files Advocacy/Managed-Care/ Guidelines-for-Imaging-Clinical-Decision-Support-Systems. pdf. Accessed July 15, 2018

14. Choi MY, Baek JH, Choi SJ, Jo AJ, Choi JA, Jung SE, et al. Development of evidence-based clinical imaging guidelines: to supply the evidence for appropriateness of diagnostic imaging studies and radiation exposure levels of patients. NECA Web site. https://www.neca.re.kr/lay1/program/ S1T11C145/report/view.do?seq=215. Published August 31, 
2016. Accessed July 15, 2018

15. Jung SE, Baek JH, Jeong WK, Yong HS, Choi MY, Ha EJ, et al. 2017 Clinical Imaging Guidelines for Justification of Imaging Study. Cheongju: KCDC, 2017

16. Balasubramanee V, Wimalasena C, Singh R, Pierce M. Twitter bootstrap and AngularJS: frontend frameworks to expedite science gateway development. 2013 IEEE International Conference on Cluster Computing (CLUSTER);2013 September 23-27; Indianapolis, IN, USA

17. Moroney L. The firebase realtime database. In: Moroney L, ed. The Definitive guide to firebase: build Android apps on Google's mobile platform. Berkeley, CA: Apress, 2017:51-71

18. Ooms J. The jsonlite package: a practical and consistent mapping between JSON data and R objects. Cornell University Library Web site. https://arxiv.org/abs/1403.2805. Accessed March 12, 2014

19. Choi SJ, Jeong WK, Jo AJ, Choi JA, Kim MJ, Lee M, et al. Methodology for developing evidence-based clinical imaging guidelines: joint recommendations by Korean Society of Radiology and National Evidence-Based Healthcare Collaborating Agency. Korean J Radiol 2017;18:208-216

20. Cooke R. Utilization management and ACR select. Radiol Manage 2015;37:9-12

21. Huber TC, Krishnaraj A, Patrie J, Gaskin CM. Impact of a commercially available clinical decision support program on provider ordering habits. J Am Coll Radiol 2018;15:951-957

22. ESR iGuide. Clinical Decision Support using European Imaging Referral Guidelines. European Society of Radiology Web site. https://www.myesr.org/esriguide. Accessed July 15, 2018

23. Remedios D, Hierath M, Ashford N, Cavanagh P, Grenier PA, Lloyd CM, et al. European survey on imaging referral guidelines. Insights Imaging 2014;5:15-23

24. Rosenthal DI, Weilburg JB, Schultz T, Miller JC, Nixon V, Dreyer $\mathrm{KJ}$, et al. Radiology order entry with decision support: initial clinical experience. J Am Coll Radiol 2006;3:799-806

25. Berner ES. Clinical decision support systems: theory and practice, 2nd ed. Birmingham, AL: Springer, 2006:3-43 